JAFAROV H.R. ${ }^{1}$, GASIMOV K.G. ${ }^{2 \varpi}$

${ }^{1}$ Khazar University,

Azerbaijan, AZ-1009, Baku, Mahsati str., 11, e-mail: huseyncafarov@gmail.com

${ }^{2}$ Institute of Biophysics, Azerbaijan National Academy of Sciences,

Azerbaijan, AZ-1141, Baku, Z. Khalilov str., 117, e-mail: gasimovk@yahoo.com

gasimovk@yahoo.com, (994-50) 424-2258

\title{
EXPRESSION PATTERN OF SIWRKY33 AND SIERF5 IN TOMATO PLANTS UNDER ELEVATED SALT CONCENTRATION AND WATER DEFICIT
}

WRKY and AP2/ERF gene families are important superfamilies involved in plant responses to the effect of environmental stressors. WRKY family are involved in a signaling system related to abscisic and salicylic acids [1], as well as signals linked to DREB genes [2] and AP2/ERF family of transcription factors are involved in signaling systems linked ethylene, salicylic acid and jasmonate $[3,4]$.

The members of ERF family TFs discovered in many monocots and dicots are divided into five subfamilies by number of their ERF domain. The members of each subfamily are involved in the wide variety of functions including developmental processes, responses to variety of biotic and abiotic stresses [5-7]. The numerous genes coding DRE/CRT binding proteins from variety of species, such as Arabidopsis DREB/CBFs [8, 9], maize $\mathrm{ZmDBF}[10]$, Brassica napus BnCBFs, barley HvCBF1 [11] were already cloned. All DREB1/CBFs contain AP2/ERF DNT binding domain that bind to CRT/DRE $[12,13]$. Neither CBF nor COR genes are expressed in normal condition. But in a cold condition $\left(4^{\circ} \mathrm{C}\right)$ the early induction of the expression of $\mathrm{CBF}$ genes are followed by expression of CBF regulated genes [9].

WRKY is one of the largest families of TFs in plants. Each WRKY contains at least one DNAbinding domain called WRKY, which consists of about 60 amino acid residues. An important feature of this domain is to have well preserved WRKYGQK heptapeptide and subsequent noncanonical zinc finger motif in its C-terminal domain [14]. WRKY TFs are widely distributed in many different kinds of plants, and its characteristics in each species vary greatly. Many WRKY TFs were identified in Arabidopsis, rice, potato, maize, tobacco [15-18]. The studies have shown that WRKY genes in C3 plants are activator of abscisic acid induced signaling pathway [19]. In Arabidopsis overexpression of AtWRKY25 or AtWRKY33 resulted in increased tolerance against salinity [20]. The studies with mutants of AtWRKY25 indicated that this transcription factor is involved in response to heat stress [2].

Despite the fact that tomato is one of economically important crops, and its genome is almost sequenced, but only some functional WRKY and ERF/AP2 TFs has been reported, and they have not been deeply studied sufficiently. Our recent studies have shown that analogous gene of WRKY33 in tomato plants, SIWRKY33 is overexpressed at elevated salt concentration and water deficit [21]. In present studies we examine the tissue-specific expression of SIWRKY33 from WRKY superfamily and SIERF5 from ERF/AP2 family in tomato plants.

\section{Materials and methods}

Plant material. Wild type tomato seeds were sterilized in $7 \%$ sodium hypochlorite solution, and washed out with double distilled water. Then the seeds were placed on two layers of the moistureed filter paper and incubated in the dark at $25^{\circ} \mathrm{C}$ for 3 days. The seedlings were transferred to nutrient solution $0.1 \times \mathrm{MS}$ and were grown hydroponically in a cultivation chamber at $25^{\circ} \mathrm{C} \pm 2^{\circ} \mathrm{C}$ in the intensity of light $150 \mu \mathrm{E} \mathrm{m}^{-2} \mathrm{~s}^{-1}$ with $15 / 9$ hours of light/dark photoperiod.

Total RNA fraction was isolated from 200 mg of the shoot apical part of plant using Tri Reagent (MRC, USA) according to the manufacturer's prescription. cDNA was synthesized by reverse transcriptase (RT-PCR) on isolated total RNA as a template followed by a PCR reaction. For RT-PCR "Quantitative RT-PCR ReadyMix kit" was used (Sigma, USA).

Cloning and subcloning of SlWRKY33 and SIERF5. The amino acid sequence of WRKY33 of Arabidopsis (GenBank accession number: NP_181381.2) was used as query sequence for the performing database searching of EST tomato

(C) JAFAROV H.R., GASIMOV K.G. 
(http://www.ncbi.nlm.nih.gov/dbEST). In accordance with the intended assembled sequence SIWRKY33 gene specific primers FL-FW (direct) and FL-RW (reverse) (Table) were designed to amplify the full-length cDNA template of SIWRKY33 mixtures obtained by RT-PCR. The open reading frame (ORF) of SIERF5 gene according NCBI accession number NM_001247583.1 was obtained by RT-PCR amplification from total RNA fraction and following PCR reaction using ERF5-FW and ERF5-RW primers. The amplified PCR products were cloned into pBluescript SK(-)vector (Agilent Technologies, USA) under the restriction sites Hind-III and BamH-I and sequenced. The ORF portion of SIWRKY33 gene was subcloned into the expression vector $\mathrm{pET}-15 \mathrm{~b}$ (Novagen, USA). For subcloning the ORF portion was amplified by means of PCR reaction using primers ORF-FW ORF-RW (Table). The primers used in this study were produced by "Integrated DNA Technologies, Inc." (USA).

Table. The sequence of primers used in cloning experiments

\begin{tabular}{|c|c|c|}
\hline Title & Sequence of primers $\left(\mathbf{5}^{\prime} \mathbf{-} \mathbf{3}\right.$ ) & Application \\
\hline FL-FW & TAGCTAAAGCTTATAAAATCTAAGTACTCATC & Retrieving full-length \\
FL-RW & CTATAGGGATCCACACGAAAAAAAATATATCAAC & cDNA of SIWRKY33 \\
\hline ORF-FW & CATGTACATATGGCTTCTTCAGGTGGAAATATG & Obtaining ORF from \\
ORF-RW & GATATCGGATCCTCAGTTAAGGAAAGAGCTGAAG & SIWRKY33 cDNA \\
\hline ERF5-FW & CATGTACATATGGGTTCTCCACAAGAGAC & Obtaining ORF from \\
ERF5-RW & GATATCGGATCCTTATATCATAACAAGCTGAGA & SlERF5 cDNA \\
\hline
\end{tabular}

Northern blotting hybridization of SIWRKY33 and SIERF5 RNAs were done on total RNA fraction, isolated from plant samples exposed to stress factors in different length of time. Digoxigenin (DIG)-labeled probes for hybridization were synthesized on cDNA template containing nucleotide sequence from start codon to end of the first WRKY domain of SIWRKY33 and from start codon to the end of AP2/ERF domain of SIERF5 genes. The process of labeling was conducted using DIG Probe Synthesis Kit (Roche USA).

Preparation of total protein for immunoblotting from different tissues were prepared with some modification of EZ buffer systems [22], and $15 \mu \mathrm{g}$ of total protein was loaded into each well of PAAG. The separated proteins were transferred from PAAG to PVDF membrane. The protein transferred membranes were hybridized against SIWRKY33- and SIERF5-specific polyclonal rabbit antibody. The visualization of hybridization was done by "ECL Western Blotting Detection" reagent (Amersham Biosciences) with the company provided method.

Stress Treatment was done on eight weeks of age of plants. The seedlings were placed in $0.1 \times$ MS nutrient solution supplemented with PEG 6000 or $\mathrm{NaCl}$ up to a final concentration of $20 \%$ and $250 \mathrm{mM}$, respectively. Samples for RNA isola- tion were taken at $0,1,6,12$ and 24 hours after treatments.

\section{Results and discussion}

Putative sequence of 1938 bp full-length cDNA of SIWRKY33, and the ORF of 1590 bp that encodes a protein of 529 amino acid residues was cloned as indicated in [21]. The ORF of SIERF5 according NCBI accession number NM_001247583.1 was obtained by RT-PCR amplification from total RNA fraction.

Northern RNA hybridization. To study the expression of SIWRKY33 gene at exposition to environmental stresses, the Northern RNA hybridization was used. At high salt stress, transcripts SIWRKY33 began to increase significantly after 1hour exposition (Fig. $1 \mathrm{~A}$, a). At $12^{\text {th }}$ hours of exposure, the expression of gene increased almost to the maximum. The observed pattern of expression was similar to that of AtWRKY33, which at 6 hours treatment the $\mathrm{NaCl}$ caused approximately a 30 -fold increase in transcripts [20].

The pattern of expression of SIWRKY33 at the exposure of plants to water deficit is same as in salt concentration but in lesser extend (Fig. $1 \mathrm{~A}, \mathrm{~b}$ ). At the first hour of exposure to water deficit the hybridization band of SIWRKY33 was not differed from the control plants, and then it started gradually to increase and reached to its maximum at $24 \mathrm{hrs}$ of treatment with water deficit. 

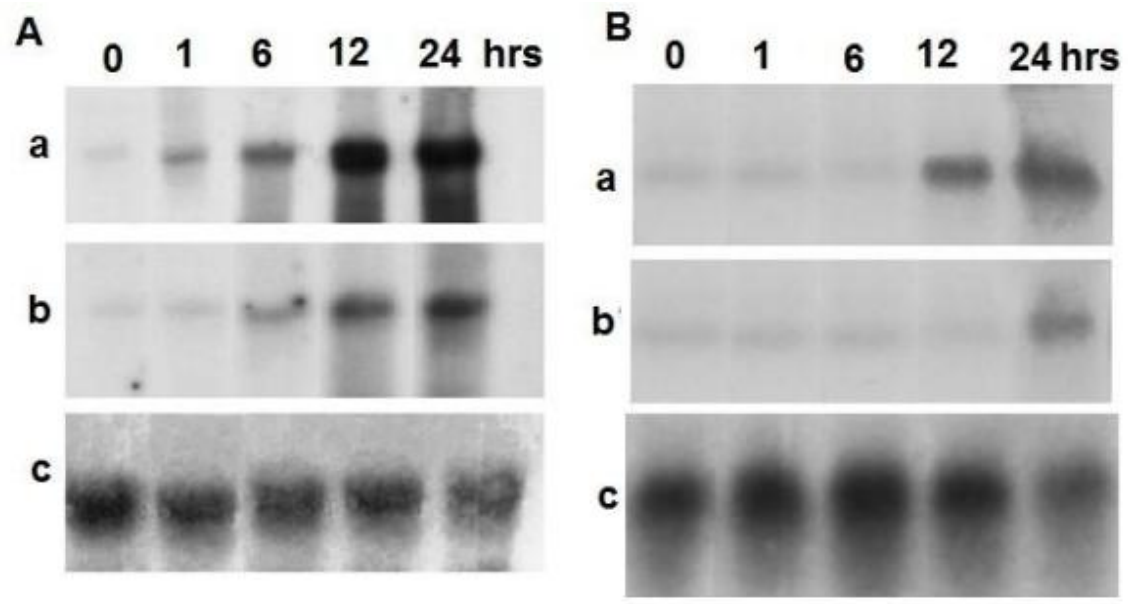

Fig. 1. Northern hybridization of total RNA fractions. A, DIG-labeled probes for hybridization prepared on SIWRKY33 sequence. B, DIG-labeled probes for hybridization prepared on SlERF5 sequence. a, RNA of plants exposed to elvated $\mathrm{NaCl}$ concentration; $\mathbf{b}$, RNA of plants exposed to water deficit. $\mathbf{c}$, control hybridization of membranes with Solanum lycopersicum actine-41 probes after removing of SIWRKY33 or SIERF5 probes. 0 - control, 1, 6, 12 and 24 , duration of plant exposure to stress conditions in hrs.

The hybridization with DIG-oxigenin labeled SIERF5 DNA probes revealed weak signals in comparison with SIWRKY3. The treatment with elevated salt concentration induced the expression of SIERF5 only at $12^{\text {th }}$ hrs of treatment (Fig. 1 B, a). But for obtaining hybridization signal with water deficit at least $24 \mathrm{hrs}$ of treatment was required (Fig. 1 B, b), moreover detected signal was weaker than that in salt stress.

Expression patterns of SlWRKY33 and ERF5 in variety of tissues of tomato

In the next step of the screening expression of SlWRKY33 the western blotting hybridization of SIWRKY33 protein with specific antibody was used. The proteins fractionated on $12 \%$ PAAG electrophoresis and transferred on PVDF membrane were hybridized against SIWRKY33-spesific polyclonal rabbit antibody. As depicted on the Fig. 2, the hybridized target protein detected on $\sim 60 \mathrm{kDa}$ region that matches to the molecular size of SIWRKY33 (58.6 kDa). This hybridization profile of SIWRKY33 protein indicates their intensive expression in variety of tissues. Among of used tissues, SIWRKY33 protein is more intensively expressed in young leaves and shoot apex and lesser extend expression is observed in stem, petiole and pedicel. Although axillary meristems are also growing point for sympodial plants like tomato no expression is observed in axillary meristems, roots and flowers.

The expression of ERF5 in tomato plant was examined by western blotting hybridization. For hybridization total protein fraction was extracted from roots, stem, flowers, pedicel, leaves, petiole, shoot apex and axillary meristems tissues of tomato plants exposed to water deficit. Proteins transferred to PVDF membrane were hybridized against SIERF5-specifik antibody. ERF5-spesifik rabbit polyclonal antibody was created on synthetic antigen designed on AP2 domain of ERF5 protein.

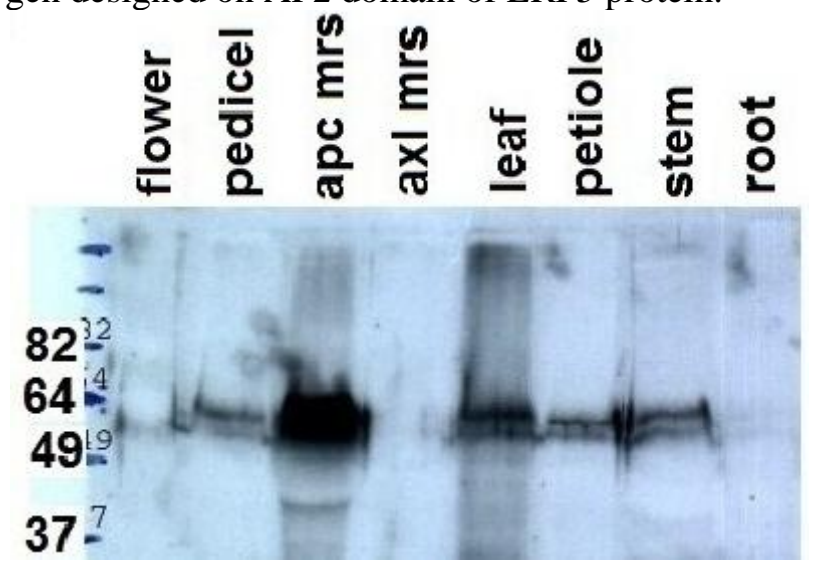

Fig. 2. Western blotting of SIWRKY33 protein with specific antibody. $15 \mu \mathrm{g}$ of total protein from variety of tissues of tomato plant exposed water deficit were loaded on SDS PAAGE. Protein transferred membrane hybridized against SIWRKY33 - specific polyclonal rabbit antibody. Marker protein's size indicated in $\mathrm{kDa}$. apcl mrs - shoot apex (apical meristems); axl. mrs. - axillary meristems.

Four tissues among of used 8 tissues - roots, stem, leaves, and shoot apex have shown intensive hybri- 
dization pattern with antibody (Fig. 3) in the region of approximately $30 \mathrm{kDa}$. Proteins from other tissues, such as axillary meristem, flowers and pedicel have shown no hybridization signals with ERF5specific antibody.

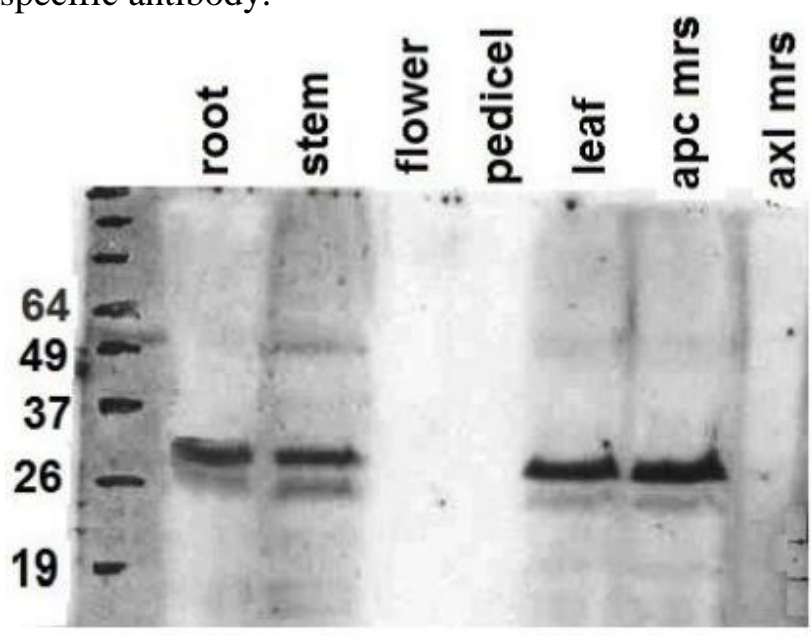

Fig. 3. Western blotting hybridization of ERF5 protein with spesific antibody. $15 \mu \mathrm{g}$ of total protein from variety of tissues of tomato plant exposed water deficit were loaded on SDS PAAGE. Protein transferred membrane hybridized against SIERF5-specific polyclonal antybody. Marker protein's size indicated in $\mathrm{kDa}$. apcl mrs - shoot apex (apical meristems); axl. mrs. - axillary meristems.

Apparently, tissue-specific expression of SIWRLY33 and SIERF5 factors in tomato plant depends on the effects of surrounding environment of plant. The involvement of these factors to abscisic acid (ABA) and salicylic acid signaling pathways was confirmed [3,4]. In turn, ABA is involved in responses of plants to drought and salt stresses [23]. The expression of the gene of abscisic aldehyde oxidase in the guard cells of Arabidopsis stomata was shown. In addition, the expression of four other genes involved ABA synthesis was also found in leaves [24], although their localization not fully discovered. But, up-regulation of ABA genes under effects of drought and elevated salt concentration is evident [25].

Based on intensity of bands obtained from hybridization in our studies, the SIWRKY33 and ERF5 (Fig. 2, 3) are expressed in leaves and apical meristems tissues that indicate intensive synthesis of signaling mediators related to the tolerance of plant. As indicated in Fig. 3, SIERF5 is intensively expressed in roots, where it can stimulate the synthesis of ABA, which is transported to leaves and other organs of plant.

Transcripts of SIWRKY33 quickly and significantly increased after exposure to salt or drought stresses (Fig. 1), it may indicate that SIWRKY33 can function through signaling pathways that control $\mathrm{NaCl}$ concentration and/or water deficit in plant cells. One such mechanism in plant organism is the regulation of ABA induced pathway, which has an interaction with DREB1B gene. It was shown that, ZmWRKY33 is induced under the influence of ABA on corn seedlings [2], it is assumed that ZmWRKY33 and DREB1B may be involved in the plant response to stress factors such as salt, drought and cold. Furthermore, our data indicate that another DREB related gene ERF5 is induced by salt concentration and water deficit, moreover its expression is induced much latter than that of SIWRKY33. Apparently, the expressed WRKY33 factor can induce the expression of ERF5 by unknown way. Therefore we do not exclude the relationship of SIWRKY33 - ERF5 /ABA DREB1B system in plant responses to salinity and water deficit.

This work was supported by Science and Technology Center of Ukraine, (STCU) Project \#6154 and partially by Azerbaijan National Academy of Sciences.

\section{References}

1. $\quad$ Yang B., Jiang Y., Rahman M.H., Deyholos M.K., Kav N.N.V. Identification and expression analysis of WRKY transcription factor genes in canola (Brassica napus L.) in response to fungal pathogens and hormone treatments // BMC Plant Biology. -2009. - 9:68. doi: 10.1186/1471-2229-9-68.

2. $\quad$ Li H., Gao Y., Xu H., Dai Y., Deng D., Chen J. ZmWRKY33, a WRKY maize transcription factor conferring enhanced salt stress tolerances in Arabidopsis // Plant Growth Regul. - 2013. - 70. - P. 207-216.

3. Grennan A.K. Ethylene Response Factors in Jasmonate Signaling and Defense Response // Plant Physiology. - 2008. - 146. P.1457-1458.

4. Lorenzo O., Piqueras R., Sanchez-Serrano J.J., Solano R., ETHYLENE RESPONSE FACTOR1 integrates signals from ethylene and jasmonate pathways in plant defense // Plant Cell. - 2003. -15. - P. 165-178.

5. Jofuku K.D., Den Boer B.G., Van Montagu M., Okamuro J.K. Control of Arabidopsis flower and seed development by the homeotic gene APETALA2 // Plant Cell. -1994. - 6, № 9. - P. 1211-1225.

6. Ohme-Takagi M., Shinshi H. Ethylene-inducible DNA binding proteins that interact with an ethylene-responsive element // Plant Cell. - 1995. - 7, № 2. - P. 173-182.

7. Okamuro J.K., Caster B., Villarroel R., Van Montagu M., Jofuku K.D. The AP2 domain of APETALA2 defines a large new family of DNA binding proteins in Arabidopsis // Proc. Natl. Acad. Sci. USA. - 1997. - 94. - P. 7076-7081. 
8. Stockinger E.J., Gilmour S.J., Thomashow M.F. Arabidopsis thaliana CBF1 encodes an AP2 domain-containing transcriptional activator that binds to the C-repeat/DRE // Proc. Natl. Acad. Sci. USA. - 1997. - 94. - P. 1035-1040.

9. Gilmour S.J., Zarka D.G., Stockinger E.J., Salazar M.P., Houghton J.M., Thomashow M.F. Low temperature regulation of the Arabidopsis CBF family of AP2 transcriptional activators as an early step in cold-induced COR gene expression // Plant J. 1998. - 16, № 4. - P. 433-442.

10. Kizis D., Pages M. Maize DRE-binding proteins DBF1 and DBF2 are involved in rab17 regulation through the droughtresponsive element in an ABA-dependent pathway // Plant J. - 2002. - 30, № 6. - P. 679-689.

11. Vernie T., Moreau S., de-Billy F., Plet J., Combier J-P., Rogers C., Oldroyd G., Frugier F., Niebel A., Gamasa P. EFD Is an ERF Transcription Factor Involved in the Control of Nodule Number and Differentiation in Medicago truncatula // The Plant Cell. - 2008. - 20. - P. 2696-2713.

12. Baker S.S., Wilhelm K.S., Thomashow M.F. The 5'-region of Arabidopsis thaliana cor15a has cis-acting elements that confer cold-, drought- and ABA-regulated gene expression // Plant Mol Biol. - 1994. - 24, № 5. - P. 701-713.

13. Yamaguchi-Shinozaki K., Shinozaki K. A novel cis-acting element in an Arabidopsis gene is involved in responsiveness to drought, low-temperature, or high-salt stress // Plant Cell. - 1994. - 6, № 2. - P. 251-264.

14. Eulgem T., Rushton P.J., Robatzek S., Somssich I.E. The WRKY superfamily of plant transcription factors // Trends Plant Sci. - 2000. - 5. - P. 199-206.

15. Zheng Z., Qamar S.A., Chen Z., Mengiste T. Arabidopsis WRKY33 transcription factor is required for resistance to necrotrophic fungal pathogens // Plant J. - 2006. - 48. - P. 592-605.

16. Journot-Catalino N., Somssich I.E., Roby D., Kroj T. The transcription factors WRKY11 and WRKY17 act as negative regulators of basal resistance in Arabidopsis thaliana // Plant Cell. - 2006. - 18. - P. 3289-3302.

17. Kim K.C., Fan B., Chen Z. Pathogen-induced Arabidopsis WRKY7 is a transcriptional repressor and enhances plant susceptibility to Pseudomonas syringae // Plant Physiol. - 2006. - 142, - P. 1180-1192.

18. Knoth C., Ringler J., Dangl J.L., Eulgem T. Arabidopsis WRKY70 is required for full RPP4-mediated disease resistance and basal defense against Hyaloperonospora parasitica // Mol Plant Microbe Interact. - 2007. - 20. - P. 120-128.

19. Zou X. et al. A WRKY gene from creosote bush encodes an activator of the abscisic acid signaling pathway // J. Biol. Chem. 2004. - 279. - P. 55770-55779.

20. Jiang Y., Deyholos M. Functional characterization of Arabidopsis NaCl-inducible WRKY25 and WRKY33 transcription factors in abiotic stresses // Plant Molecular Biology. - 2009. - 69, № 1. - P. 91-105.

21. Gasimov K.G., Najafova L.A. SIWRKY33 promotes tomato plant tolerance to salt and drought stresses // Ukrainian Journal of Plant physiology and Genetic. - 2014. - 46. - № 5. - P. 385-394.

22. Martínez-García J.F., Monte E., Quail P.H. A simple, rapid and quantitative method for preparing Arabidopsis protein extracts for immunoblotting analysis // Plant J. - 1999. - 20, № 2. - P. 251-257.

23. Yokota A., Takahara K., Akashi K. Water stress. Physiology and molecular biology of stress tolerance in plants. Edited by K.V. Madhava rao, A.S. Raghavendra, K. Janardhan Reddy. - Springer. - 2006, - P. 15-40

24. Tan B.C., Joseph L.M., Deng W.T., Liu L., Li Q.B., Cline K., MaCarty D.R. Molecular characterization of the Arabidopsis 9cis expoxycarotenoid dioxygenase gene family // Plant J. - 2003. - 35. - P. 44-56.

25. Assmann S.M., Snyder J.A., Lee Y.-R.J. ABA-deficient (abal) and ABA-insensitive (abil-1, abi2-1) mutants of Arabidopsis have a wild-type stomatal reponse to humidity // Plant Cell Environ. - 2000. - 23. - P. 387-395.

JAFAROV H.R. ${ }^{1}$, GASIMOV K.G. ${ }^{2}$

${ }^{I}$ Khazar University, Azerbaijan, AZ-1009, Baku, Mahsati str., 11, e-mail: huseyncafarov@gmail.com

${ }^{2}$ Institute of Biophysics, Azerbaijan National Academy of Sciences, Azerbaijan, AZ-1141, Baku, Z. Khalilov str., 117, e-mail: gasimovk@yahoo.com

\section{EXPRESSION PATTERN OF SIWRKY33 AND SIERF5 IN TOMATO PLANTS UNDER ELEVATED SALT CONCENTRATION AND WATER DEFICIT}

Aim. High salinity, drought, and low temperature are the major abiotic stresses affecting plant growth and development and can lead to serious yield losses of agricultural crops. In plants, the majority of responses to abiotic stresses are controlled at the transcriptional level that is regulated by transcription factors (TFs). The family of WRKY and AP2/ERF are important superfamilies involved in response of plants to abiotic stress. Methods. The tissue-specific expression pattern of two superfamily members - SIWRKY33 from WRKY and SIERF5 from AP2/ERF were studied in tomato plants by means of Northern RNA hybridization and immunoblotting of total proteins. Results. Northern hybridization revealed very quick and strong expression of SIWRKY33 at elevated concentration of salt and water deficit, and relatively late and weaker expression of SIERF5 genes. Western hybridization with specific polyclonal antibodies revealed that during water deficit SIERF5 and SIWRKY33 were intensively expressed in variety of tissues of tomato plants. SIERF5 expressed in shoot apex, young leaves, roots and stems, while SIWRKY33 expressed in the same tissues as above and in axillary buds. Conclusions. Obtained results indicate that both SIWRKY33 and SIERF5 are involved in plant responses to abiotic stresses like elevated salt concentration and water deficit.

Keywords: Solanum lycopersicum L., transcription factor, cloning, immunohybridization abiotic stresses. 